\title{
Mobile Surveillance using Wireless Technology
}

\author{
V.Venmathi \\ Assistant professor \\ SNS College of Engineering \\ Coimbatore
}

\author{
R.Sripriya \\ UG scholars \\ SNS College of Engineering \\ Coimbatore
}

\author{
L.Kiruthika, \\ UG Scholars \\ SNS College of Engineering \\ Coimbatore
}

\author{
P.Megala \\ UG Scholars \\ SNS College of Engineering \\ Coimbatore
}

\begin{abstract}
The goal of this project was to design a quadcopter which is used for System identification. The surveillance operator uses a sophisticated electronic equipment to find, sort and identify within a defined region. Here, the Quadcopter is designed with rotors and an Arduino processor for system identification. The required information from the quadcopter is being transmitted using a Zigbee module. A flying Quadcopter is designed in such a way the altitude is measured and controlled using an electronic speed controller which is connected to a DC motor. The abnormalities detected using the webcam are carried onto the receiver side for surveillances. The structure of the quadcopter is designed using an AutoCADD software.
\end{abstract}

\section{Keywords}

Quadcopter, Arduino processor, Zigbee, AutoCADD

\section{INTRODUCTION}

Accurate dynamic modeling of helicopter aeromechanics is becoming increasingly important for System identification [15]. The surveillance operator uses a sophisticated electronic equipment to detect, classify and identify within a specified area. Here, the design of Quadcopter with rotors for system identification with an Arduino processor. In order to fly the quadcopter the required information from the quadcopter is being transmitted using a Zigbee module (CC2500) [10]. RF Module is a transceiver module which helps to access RF communication at $2.4 \mathrm{GHz}$ and it is used to transmit and receive the data at 9600 baud rates. It provides extensive hardware support for packet handling, data buffering, burst transmissions, clear channel assessment, link quality indication and work on the radio. A flying Quadcopter is designed in such a way that the altitude is measured and controlled using an Electronic Speed Controller (ESC). The signals to the ESC's are directly sent by the transmitter remote control through the receiver. The transmitter is used by the user to control the quad. If the user changes the input, the transmitter sends radio signals to receiver and receiver changes these signals to PWM signals [6]. Arduino processor is used for converting the radio signals into PWM signals. These PWM signals are sent to ESC's causing the change in the speed of the motor. The speed of the motor is controlled [12] in such a way that the hovering of the quad is controlled. Here, Brushless DC motor is used because of its good speedtorque characteristics [7], [15] and low maintenance. All the motors are connected to Electronic speed controllers (ESC) to control the speed of the rotors which in turn supplies motor with the required power. Each rotor produces a thrust and a torque about its center of rotation. The propellers (P1, P4) mounted on opposite arms are set in one direction and the propellers (P2, P3) are set in the opposite direction, the forces

\author{
C.Subbulakshmi \\ UG scholars \\ SNS College of Engineering \\ Coimbatore
}

produced by it helps the quadcopter to fly. Lithium-ion polymer battery provides power to all individual circuits and it also supplies the operating voltage $5 \mathrm{~V}$ to the Arduino board. The abnormalities are detected using the webcam and then it is carried onto the receiver side for surveillance and control. Here, the vertical takeoff [17], [19] and landing of the quadcopter are controlled by the user manually. The software, "Arduino", can be used to program the Arduino microcontrollers. The main part of the program is PID algorithm. It is used to minimize the error between the target and the current position [9]. The structure of the quadcopter is designed using an AutoCADD software. The Quadcopter is sent to the spot for live telecast [11], [13], [14] and it is used for defense purposes.

\section{2. BLOCK DIAGRAM}

The Figure 1 shows the Block Diagram of the Transmitter side it consists of Zigbee (CC2500), RF tuner, Television, Power Supply and Camera.
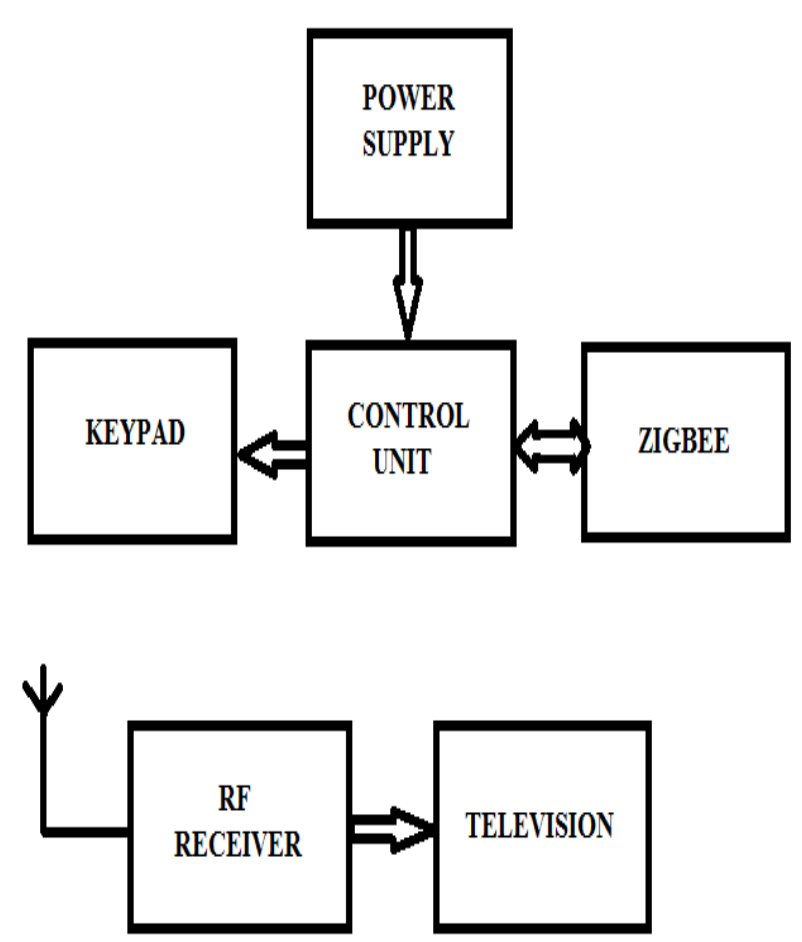

Figure 1: Transmitter side

Figure 2 shows the Receiver side. It consists of Arduino Processor (AT Mega 2560), Brushless DC Motor (A2212), Electronic Speed Controller, Propeller, Zigbee (CC2500), and Power Supply. 


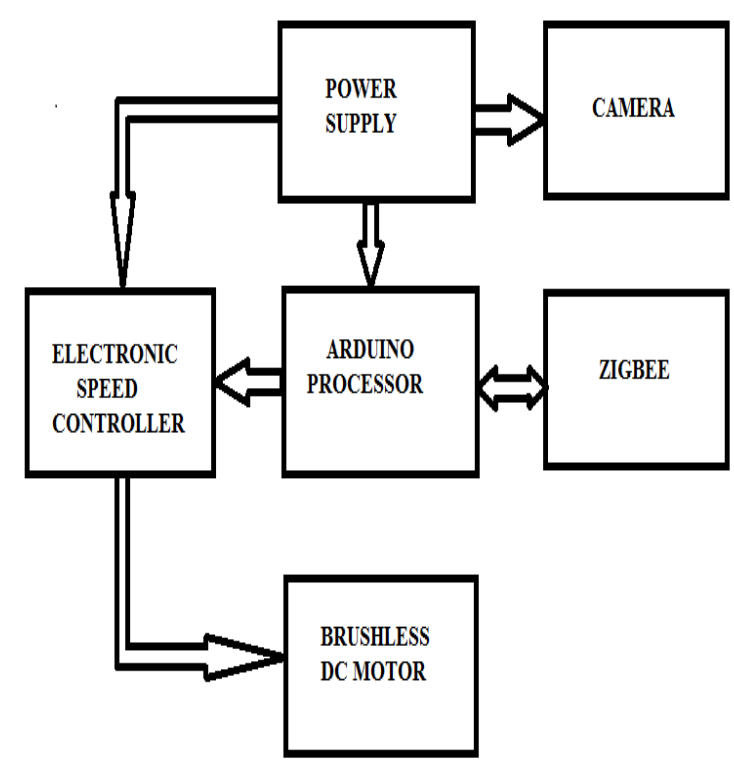

Figure 2: Receiver side

A quadcopter is a helicopter which has four equally spaced rotors, usually arranged at the corners of a square body. The rotors are used to push the air upwards and downwards in order to keep the quad aloft. In [2] the digital control of brushless DC motor was discussed. A brushless motor is constructed with a permanent magnet rotor and wire wound stator poles. Electrical energy is converted to mechanical energy by the magnetic attractive forces between the permanent magnet rotor and a rotating magnetic field induced in the wound stator poles. There are three electromagnetic circuits connected at a common point. Each electromagnetic circuit is split in the center, thereby permitting the permanent magnet rotor to move in the middle of the induced magnetic field. They are most popular in industrial and actuation application. Here the rotor position is sensed by the Hall Effect sensor [1], [17]. The Propellers are connected to each rotor in the brushless DC motor. ESC [10] is directly connected with the brushless DC motor. It consists of three wires such as PWM, positive and ground. The PWM signals are applied to the inverter circuit (ESC) at the appropriate time to trigger the switches and this signal is used to control the motor. As soon as the power supply is ON, the ESC creates beep sound and this indicates the throttle setting of the motor. Here, we use lithium ion polymer battery [10] because of its long life span. The lithium ion polymer battery is thin, light and powerful. The output voltage is $7.4 \mathrm{~V}$ and storing charge is $2200 \mathrm{mAh}$. A lithium ion polymer battery is a fast growing and most emerging battery. It has light weight, high density and low maintenance. The battery is connected to all Hardware modules. In our setup we use two zigbee modules. One is connected to the Arduino processor board on the Quadcopter while the other one is connected to a computer on the ground and it acts as both transmitter and receiver. Zigbee [10] is targeted at applications that require a low data rate, long battery life, and secure networking. Zigbee Module is a transceiver module which provides easy to use RF communication at $2.4 \mathrm{GHz}$. It can be used to transmit and receive data at 9600 baud rates from any standard Complementary MOSFET (CMOS) source. It is used in both transmitter and receiver side to transfer the information. The required input voltage is $5 \mathrm{~V}$. When Zigbee transmits the data to the control unit, then the motor start to rotate. The commands UP, DOWN, RIGHT, LEFT is used to make the Quad fly. These commands are programmed in the Arduino Processor [9], [16] using Arduino software. The development of quadcopters has stalled recently, because controlling four independent rotors have proven to be incredibly difficult and impossible without electronic assistance. The decreasing cost of the processors and controllers has made the electronic and even completely autonomous control of quadcopters feasible for commercial, military, and even hobbyist purposes. And the surveillance system that can be provided in case of a hazard when communication has been cut down [11].We designed the Quad structure using AutoCADD Software which is shown in Figure.3

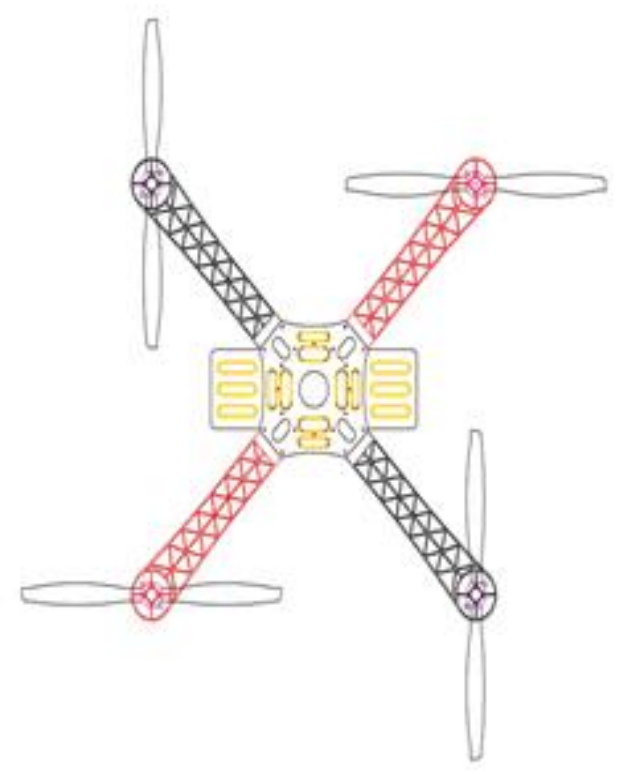

Figure 3: Quad structure

\section{HARDWARE MODULES}

\subsection{Arduino Processor}

We have chosen the Arduino processor [9], [16] because it simplifies the amount of hardware and software development. The Input voltage required for the processor is $7-12 \mathrm{~V}$ and it limits up to $6-20 \mathrm{~V}$. The operating voltage is $5 \mathrm{~V}$. The Arduino Mega can be powered via the USB connection or with an external power supply. The power source is selected automatically. The digital pin D9, D10, D11, D12 are connected to the ESC of each motor which is used to control the speed based on the switching time. The Arduino generates the PWM signals and it is given to the ESC to control the speed of the motor. The TX and RX pins on the Arduino are connected to the Zigbee [10], which is used to transmit and receive the information. The Arduino software provides the number libraries which makes the programming of the microcontroller easier. By dumping the program in the arduino we can able control the direction of the Quad.

\subsection{Propeller}

A propeller is a type of fan that transmits power by converting rotational motion into thrust. The propellers are designed in such a way that two propellers push the air in one direction and other two propellers push the air in the opposite direction. So, that the torque produced by the rotors get cancelled and make the structure fly at greater distance. We have used 2 blades, $9 \times 4.7$ pitch which can transfer more energy onto the air, thus providing more lift. 


\subsection{Electronic Speed Controller}

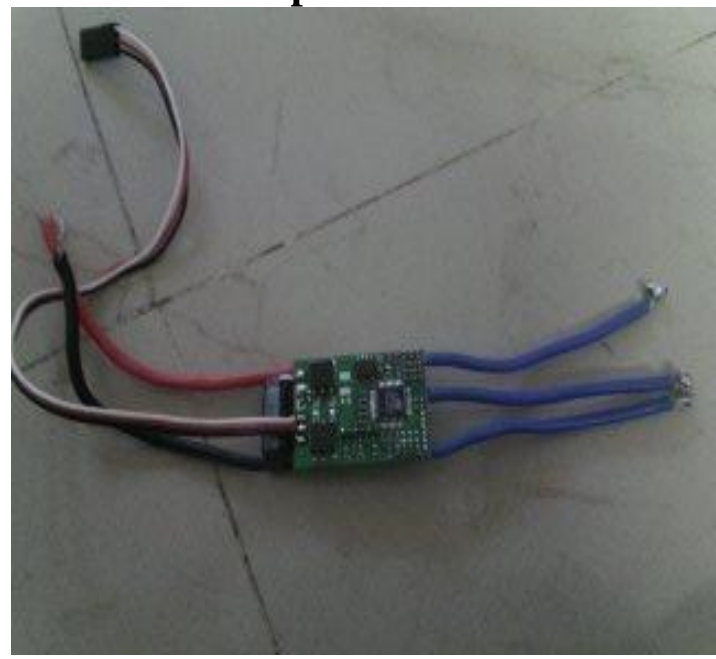

Figure 4: ESC

An electronic speed control shown in the Figure 4 ESC is an electronic circuit with the purpose to vary an electric motor's speed, its direction and possibly also to act as a dynamic brake. ESCs are often used on electrically powered radio controlled model, with the variety often used for brushless motor essentially providing an electronically-generated three phase electric power low voltage source of energy for the motor. A brushless AC motor controllers are much more complicated than brushed motor controllers. An ESC [10] has three sets of wire Red, Black, White. The Red wire indicates the positive supply. The black wire indicates the ground. The White wire indicates the PWM signals. In modern speed controller where the three wires for the ESC is of the same color, attach any three wires and to turn the motor direction around swap the black and yellow motor cables around. The controller works by switching the battery connection to the motor on and off around 20,000 times a second. The motor averages this out, as this rate of switching is too fast for the motor to detect. If the battery is only connected for half the total time, then the motor sees the $24 \mathrm{~V}$ battery as if it were only $12 \mathrm{~V}$, and goes at half speed. Also, because the switching is so fast, the motor's inductance - which acts like an electrical flywheel - keeps the current in the motor flowing constantly. But the current only flows for half of the time from the battery, so the battery current will be half the motor current.

\subsection{Wireless Webcam}

A webcam is a video camera that feeds or streams its image in real time to or through a computer or computer network. Here, we have used is CMOS wireless camera. When images or video is captured by the camera [11], the video stream may be viewed or sent to the other networks via systems such as the internet, and email as an attachment. Wireless security cameras are CMOS cameras that is fixed in the flying setup. It requires a supply of $9 \mathrm{~V}$ so we used general purpose battery. As soon as the supply is ON, the camera captures the video. On the transmitter side we have RF receiver and TV television). By proper tuning (frequency control), the video signals are received and it can be viewed through television. The receiving frequency of the video signal is $2.4 \mathrm{GHz}$. Wireless cameras are proving very popular among modern security consumers due to their low installation costs (there is no need to run expensive video extension cables) and flexible mounting options; wireless cameras can be mounted/ installed in locations previously unavailable to standard wired cameras.

\section{SIMULATION AND RESULT}

The surveillance system developed is a road based system. Using Arduino processor, we control the whole system. The coding is developed in order to generate the PWM signals with $95 \%$ of duty cycle. With this long period of ON time, the motor is now able to rotate with high RPM. Because of the more force, the torque developed by the rotors get cancelled and it helps to lift the structure. Using Zigbee on the transmitter and receiver side the flying mode of the quad is controlled. Thus the speed of the motor is controlled at the maximum level for vertical takeoff and by using the Zigbee module the flying mode of the quad is controlled upto 100 meters. The wireless camera used here, transmit the video signal. By proper frequency tuning (using RADIO AV RECEIVER), the video is received and it can be viewed through television. With the aid of the necessary setups and CMOS camera, a live video is telecasted. Future work deals with, using of GPS control for long range communication and by using gestures the direction of the quadcopter is controlled. The complete setup of our project is shown in the Figure 5.

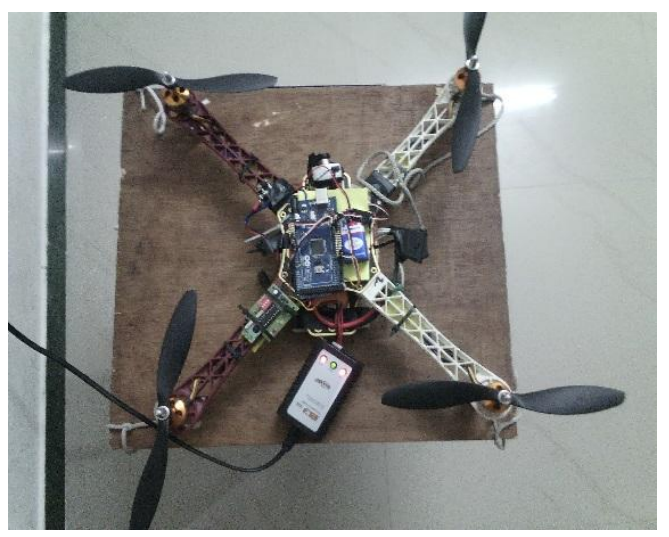

Figure 5: Hardware module

\section{CONCLUSION}

Quadcopter is a generally unmanned aerial vehicle type device which uses for applications like surveillance and for defense applications. So it can reach the places where man can't so it would be a better choice under hostile circumstances so we can use it as per the requirements while this is totally controlled by Arduino controller. It generates PWM signal to control the speed of the motor which is connected to the Arduino via ESC. So, basically Quadcopter is such type of device which is same as drones, so we can use this under unpleased environmental conditions.

\section{REFERENCES}

[1] Kartik Agarwal, Karthik Korada, Piyush Sahoo, Rushikesh Chaudhari, Vikram Singh "Autonomous Quadcopter".

[2] C. Sheeba Joice, S. R. Paranjothi, V. Jawahar Senthil Kumar "Digital Control Strategy for Four Quadrant Operation of Three Phase BLDC Motor With Load Variations", IEEE Transactions on INDUSTRIAL INFORMATICS, VOL.9, NO.2, MAY 2013.

[3] Young Jung, Yong-Jae Kim, Jungmoon Jae, Jaehong Kim "Commutation Control for the Low-Commutation Torque Ripple in the Position Sensorless Drive of the Low -Voltage Brushless DC Motor", IEEE Transactions on POWER ELECTRONICS, VOL.29, NO.11, NOVEMBER 2014. 
[4] Xiaohong Nian, FeiPeng, Hang Zhang "Regenerative Braking System of electric Vehicle Driven by brushless DC Motor", IEEE Transactions on INDUSTRIAL ELECTRONICS, VOL.61, NO.10, OCTOBER 2014.

[5] Jian Shi and Tie-CAI Li "New method to eliminate commutation torque ripple of brushless DC moor with minimum commutation time" IEEE Transactions on INDUSTRIAL ELECTRONICS, VOL.60, NO.6, JUNE 2013.

[6] Vashist Bist, Bhim Singh, "A PFC-Based BLDC Motor Drive Using a Canoncal Switching Cell Converter", VOL 10, NO 2, MAY 2014.

[7] Neena Mani, Ammu Catherine Treesa, Anju Sivadas, Celus Sheena Francis, Neethu M.T, "Visual Tracking and Surveillance System”, VOL 3, MAY 2014.

[8] Vashist Bist, Bhim Singh "An adjustable-speed PFC bridgeless buck-boost converter -fed BLDC motor drive" IEEE Transactions on INDUSTRIAL ELECTRONICS, VOL.61, NO.6, JUNE 2014.

[9] Li Wang, Senior Member, IEEE, and Dong-Jing Lee "Coordination, Control of an AC-to-DC Converter and a Switched Excitation Capacitor Bank for an Autonomous Self-Excited Induction Generator in Renewable-Energy Systems" IEEE Transactions on INDUSTRY APPLICATIONS, VOL. 50, NO. 4, JULY/AUGUST 2014.

[10] Mustafa Mukadam, Christopher Moy and Silver De Guzmen “Alternative Quadcopter” 2013.

[11] Xinxiu Zhou, Jiancheng Fang, "Precise Braking Torque control for Attitude Control Flywheel With Small Inductance Brushless DC Motor', VOL 28, NO 11, NOV 2013

[12] Kyung-Tae Kim, Jun-Kyu Park, Jin Hur, Senior Member, IEEE, and Byeong-Woo Kim "Comparison of the Fault Characteristics of IPM-Type and SPM-Type
BLDC Motors Under Inter-Turn Fault Conditions Using Winding Function Theory, IEEE Transactions on INDUSTRY APPLICATIONS, VOL. 50, NO. 2, MARCH/APRIL 2014

[13] L.R Garcia Carrillo, A. Dzul and R. Lozano "Hovering quad-rotor control: A comparison of non linear controllers using visual feedback "IEEE Transactions on AEROSPACE AND ELECTRONIC SYSTEM VOL.48, NO.4 OCTOBER 2012.

[14] Adib Khozouhee, Christopher Brennan, Edmar Goncalves, Ejiroghene Urhiafe, Andras Moritz, Roderick Grupen "Automated Aero-painting System".

[15] Marco Bergamasco and Marco Lovera "Identification of linear models for the dynamic of a hovering quadrotor" IEEE Transactions on CONROL SYSTEMS TECHNOLOGY, VOL. 22, NO.5, SEPTEMBER 2014

[16] Kenneth D. Sebesta and Nicolas Boizot, "A Real-Time Adaptive High-Gain EKF, Applied to a Quadcopter Inertial Navigation System",IEEE Transactions on INDUSTRIAL ELECTRONICS, VOL 61, NO 1, JAN 2014.

[17] Abdelhamid Tayebi and Stephen McGilvray "Attitude stabilization of a VTOL Quadcopter Aircraft" IEEE Transactions on CONTROL SYSTEMS TECHNOLOGY, VOL. 14, NO.3, MAY 2006.

[18] Courtney E. Doyle, Student Member, IEEE, Justin J. Bird, Taylor A. Isom, Jason C. Kallman, Daman F. Bareiss, David J. Dunlop, Raymond J. King, Jake J. Abbott, Member, IEEE, and Mark A. Minor, Member, IEEE "An Avian-Inspired Passive Mechanism for Quadrotor Perching", IEEE/ASME Transactions on MECHATRONICS, VOL, 18, NO. 2, APRIL 2013.

[19] Bruno Herisse, Tarek Hamel, Robert Mahony and Francois-Xavier Russotto "Landing a VTOL unmanned aerial vehicle on a moving platform using optical flow", IEEE Transactions on Robotics, VOL.28, No.1, FEBRU 